\title{
Preview: 2000 MRS Fall Meeting
}

\author{
Hynes Convention Center and Sheraton Boston Hotel, Boston, Massachusetts \\ NOVEMBER 27-DECEMBER 1, 2000 \\ Meeting Chairs: Sungho Jin (Bell Labs-Lucent Technologies), Antonios G. Mikos (Rice University), \\ David J. Srolovitz (Princeton University), and Bernd Stritzker (Universität Augsburg)
}

The 2000 Materials Research Society Fall Meeting will be held November 27 through December 1 at the Hynes Convention Center in Boston for the second year and the Sheraton Boston Hotel. This transition from the Marriott Hotel makes it very convenient for participants to attend different sessions because of the close proximity of the two sites. The Meeting offers 41 technical symposia that are grouped into eight distinct clusters, plus Symposium X, "Frontiers of Materials Research," as Cluster 9, which consists of special invited talks for the nonspecialist. An esoteric blend of new areas as well as established materials-research fields, the symposia topics represent current trends in interdisciplinary materials research. The thread of nanotechnology runs through a number of symposia.

Organic and biomaterials are covered under the umbrella of soft materials (Cluster 8). The use of organic and polymeric materials for electronic and photonic devices is rapidly growing in numerous applications, including light-emitting diodes, transistors, lasers, and photodiodes. Symposium JJ will address these materials and technologies, and will hold a joint session with Symposium D on molecular devices. Symposium KK will focus on filled and nanocomposite materials. Various polymer-composite materials will be covered, including filled systems, advanced nanocomposite systems, block and segmented polymer systems, and molecular composites, with an emphasis on structure-property relationships. Sessions include modeling of properties, rubber-toughened materials, materials aging, and advanced nanocomposites.

Symposia LL-OO on biomaterials have been endorsed by the Society for Biomaterials. Symposium LL will address tissue engineering and dental/maxillofacial biomaterials on Monday, orthopedic and musculoskeletal materials on Tuesday, and novel materials in the Wednesdaymorning session. Cardiovascular biomaterials are the focus of Symposium MM. Symposium NN deals with the rapidly developing field of drug delivery, highlighting devices for controlled and targeted drug delivery and materials for various delivery systems. These include stimuli-

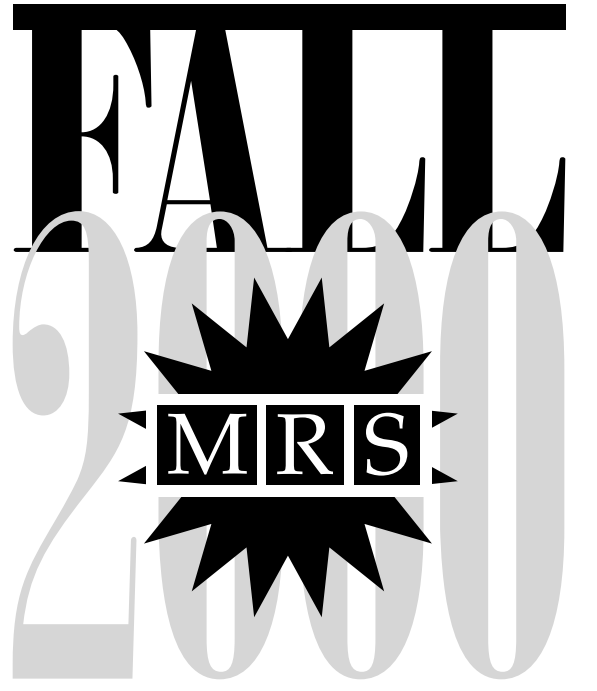

November 27 - December 1 Boston, Massachusetts

sensitive polymers, phase behavior, protein and peptide delivery, tissue and gene delivery, phase separation, and novel approaches to drug delivery systems. Neurologic biomaterials, covered in Symposium $\mathrm{OO}$, are used to assist regeneration in the central or peripheral nervous systems as a result of injury or disease.

Nanostructured materials have become an important area for study in materials science, and Cluster 1 highlights various aspects of nano- and microstructured materials. Symposium A will focus on carbon nanotubes, which hold potential for technological applications. Carbon nanotubes also represent an ideal system for studying nanometer-scale materials science. Sessions will cover synthesis, properties, hydrogen storage, composites, nanorods, tube filling, and applications, including sensors and probes.

Comparisons of theoretical studies and experimental results for structure and mechanical properties of nanophase materials will form part of Symposium B. A joint session with Symposium W is scheduled for Wednesday afternoon, with talks on the inverse Hall-Petch effect, limits of grain-refining strengthening in iron, atomistic studies of plasticity in nanophase metals, and grain-boundary-controlled deformation studies of nanocrystalline materials using molecular-dynamics simulations, among others. Nanoscale dimensions of nanoparticles give rise to anisotropy. This aspect will be explored in Symposium $\mathrm{C}$ on anisotropic nanoparticles, including synthesis, characterization, and manipulation. Of special interest is the assembly of ordered one-, two-, and three-dimensional arrays of anisotropic nanoparticles and the fabrication of new devices and structures. Symposium sessions will focus on metallic nanoparticles and clusters, nanowires, biological nanoparticles, semiconductors, and dielectric nanoparticles.

A critical step in nanotechnology is nanofabrication at submicron length scales. Fabrication techniques ranging from lithographic methods to new selforganizing approaches will be covered in Symposium D. A session on Tuesday afternoon will cover unconventional approaches to nanofabrication. Symposium $E$ will focus on the growing field of microphotonics, with its emphasis on manipulation of photons using photonic bandgap and high-dielectric-contrast optics. Symposium F will concentrate on nano- and microscale semiconductor materials and structures, continuing a series of MRS symposia in this field. Nano- and microcrystalline semiconductors already play a critical role in microelectronics, and sessions will cover various topics, including biology with nanoscale silicon, silicon nanowires, diamond nanocrystals, rare-earth materials, and light-emitting devices.

Semiconductor materials form the focus of Cluster 2. Symposium G on Group III nitrides continues a sequence of symposia in MRS meetings. The symposium starts with a tutorial on Sunday on materials characteristics of the III-nitrides. A special session is scheduled on Wednesday afternoon in honor of I. Akasaki (Meijo University) for his contributions to the field, featuring a talk by Akasaki as well as by others on optical properties of III-IV quantum structures, epitaxial lateral overgrowth of $\mathrm{GaN}$, and microwave $\mathrm{AlGaN} /$ 
GaN power HEMTs (high-electronmobility transistors). SiC-based electronic systems, the focus of Symposium H, offer enormous potential in high-voltage, hightemperature, and high-frequency applications. Reducing defect densities in bulk $\mathrm{SiC}$ crystals, controlling morphology and residual impurities in epilayers, and the development of new device structures will be addressed. Sessions on bulk growth, epitaxy, devices, implantation, and metallization are also scheduled.

Symposium I covers one of the more interesting and promising new areas of materials science research: semiconductor spintronics. The basis for this field is the use of electron spin for the storage and transport of information by manipulating the spin degree of freedom of electrons. Fundamentally new ideas and paradigms in electronics are now possible.

Semiconductor quantum dots, the focus of Symposium J, are nanometer-sized giant molecules or crystals. The chemical and physical properties of these materials vary as a function of size in accordance with scaling laws. With rapid advancements in the field - and ideally suited for scientific studies-quantum dots are beginning to make inroads into applications. Sessions address biological applications, growth processes, characterization techniques, and quantum-dots-based devices.

Inorganic materials are the focus of Cluster 7, in which concrete, solid-state chemistry, catalytic materials, and hightemperature superconductors are covered. Symposium FF provides a forum for presentations and discussions on the state of the art in newer formulations of concrete. Sessions cover microstructure and rheology, transport, mixture proportioning, mechanical properties, durability, field performance, computerized systems, and shrinkage. The goal of solid-state chemistry of inorganic materials, Symposium GG, is to understand, control, and predict structures and properties of solids at the atomic level. A special session on Monday afternoon honors J.M. Honig (Purdue University) for his contributions to the field and for his stewardship of the Journal of Solid State Chemistry. Thursday's sessions include solid-state ionics, battery materials, energy storage, thermopower, thermal expansion, and optical materials.

Advanced catalytic materials (Symposium $\mathrm{HH}$ ) are critical for the chemical and petrochemical industries, and great strides continue to be made in the development of better and newer catalytic materials. Sessions include porosity design, catalytic testing and analysis, carbon and metal carbides, clays, metal hydroxides and oxides, and nano- crystalline catalysts. In the several years since the discovery of higher-temperature superconductors (Symposium II), significant work has been done, and the present challenges include moving from the laboratory to industry. Sessions cover different aspects of coated-conductor science and technology as well as microstructural underpinnings of the properties and performance of superconductors.

Cluster 3 on metals covers an eclectic range of topics. Symposium K on quasicrystals will include theory, applications, and metallurgy. A joint session with Symposium L on glassy metallic alloys is scheduled for Wednesday morning. Other sessions are devoted to applications, including quasicrystalline coatings, thermoelectric applications, catalysis, and reinforcement for polymer composites. The scheduled sessions in Symposium L on supercooled liquid, bulk glassy, and nanocrystalline states of alloys will cover glass-forming ability and thermal stability, mechanical properties, magnetic properties, phase-diagram calculations, and the atomic and electronic structure of these materials.

Symposium $\mathrm{M}$ addresses relevant issues for thermal-barrier coatings that are used in gas-turbine engines and some diesel engines. Sessions cover failure mechanisms, lifetime predictions, mechanical properties, oxidation issues, and processing. Five plenary talks address durability issues, life predictions, failure mechanisms, and thermal-barrier coatings via electron-beam physical vapor deposition (EBPVD). Sessions in Symposium N on high-temperature-ordered intermetallic alloys are organized based on the material and cover titanium aluminides, nickel aluminides, iron aluminide, iridium, and metal silicides.

Ion-beam modification, processing, and synthesis of materials has been strongly represented at MRS meetings. Symposium $\mathrm{O}$ opens with a talk by L.C. Feldman (Vanderbilt University) on 21st-century requirements for ion-beam-materials interactions. Subsequent sessions address defect kinetics and fundamentals, as well as modification of different classes of materials using ion beams. On Wednesday, joint sessions will be held with Symposium $\mathrm{R}$ to cover nanostructures and thin layers, optoelectronics/photonics, and microelectronic materials, as well as semiconductors and other electronic materials. Symposium P will highlight growth and postgrowth evolution of ultrathin films and self-organized structures on surfaces. The symposium covers a broad range of topics including nanoscale surfaces, magnetic films, morphological evo- lution of surfaces and thin films, insulating and organic surfaces, and epitaxy.

Symposium Q, on the fundamentals of nano-indentation and nanotribology, focuses on modeling and simulations, deformation mechanisms, new measurement and analysis methods, and adhesion. A joint session with Symposium W on limits of strength in indentation will be held on Thursday morning. Radiation can cause significant changes to the microstructure of materials, leading to dramatic changes in properties, which will be addressed in Symposium R. The symposium will explore radiation effects in metals, ceramics, and electronic materials, as well as theory. Three joint sessions with Symposium $\mathrm{O}$ are scheduled for Wednesday.

An externally applied magnetic field can have significant influence on the behavior of materials. Symposium S will cover magnetic levitation, magnetic annealing, ferromagnetic shape-memory alloys, grainboundary dynamics and phase transformations, suspension of particles in a dc or ac magnetic field, magnetically structured materials, and solutions and electrodepositions in a magnetic field. Symposium T on the effects of confinement on gaseous, liquid, and solid systems includes presentations about biomolecules confined in silica nanopores, the dynamics of layering transitions of confined liquids, friction at the continuum limit, single-molecule dynamics, sliding transitions, mechanics, and dissipation in nanoscale contacts.

The availability of ultrafast, high-intensity pulsed lasers has spurred efforts in the area of the dynamics of electrons and atoms interacting with intense optical fields in various materials (Symposium U). Along with fundamental studies, the practical technological implications are significant such as in laser-induced materials processing. The focus of Symposium V is on wet and environmental scanning electron microscopy (ESEM). SEM can be performed in the "low vacuum" regime, at higher pressures than conventional highvacuum SEM. Water can be maintained in the liquid state at the upper range of this operating pressure region. This now allows for the observation of dynamic processes. D. Newbury (NIST) opens the symposium on Wednesday morning with an overview of low-vacuum SEM. E. Doehne (Getty Conservation Institute) will give a talk on the use of ESEM on stone samples from a Mayan site in Honduras. D. Joy (University of Tennessee) will talk about the use of ESEM in semiconductor technology.

Symposium $\mathrm{W}$, the first symposium in Cluster 5 , explores modeling and theory addressing the ultimate limits to 
mechanical strength. Symposium Y will examine dislocation behavior and the effects of interfacial influences on microstructural evolution in metallurgical systems. Sessions will be held on interface motion, grain motion, interface anisotropy, interfacial chemistry, dislocations and plasticity, and interface/dislocation interaction. A joint session with Symposium $\mathrm{Z}$ on strain localization and dislocation patterning is scheduled for Wednesday morning.

Symposium $\mathrm{Z}$ will tackle another aspect of modeling, namely, multiscale materials modeling. Multiscale modeling now allows for the possibility of linking atomicscale processes to macroscopic phenomena. Sessions will be held on dislocation plasticity, fracture, atomistics, polycrystalline plasticity, point defects, and thin films. Symposium AA will explore oxide surfaces and interfaces in ceramics, particularly for modeling structure-property relationships. Grain boundaries, interfaces in multilayers and composites, metal-oxide interfaces, surfaces, and oxide thin-film epitaxy will be covered.

In Cluster 6 on devices and functional materials, Symposium BB will focus on characterization and modeling of domain microstructures in materials. Sessions cover domain-related phenomena in ferroic materials and include a joint session with Symposium CC on Wednesday addressing ferroelectric thin films. Symposium CC begins with sessions on Monday to cover nonvolatile memories and integration and electrodes, and continues with a poster session that evening on barium strontium titanate (BST), gate materials and dynamic random-access memory (DRAM), integration and electrodes, and nonvolatile memories. On Tuesday, sessions will cover BST for DRAM and gate dielectrics, and fundamental properties of ferroelectric thin films. The session on Thursday afternoon will be held jointly with Symposium DD on high-frequency applications.

Also in Symposium DD on materials issues for tunable $\mathrm{rf}$ and microwave devices, S. Wolf (DARPA/DSO) will give an update on the DARPA program on frequency agile materials. S. Toncich (KWC Inc.) will present a paper on improved techniques for the measurement of loss tangent of ferroelectric thin and thick films in the microwave region. F. Miranda (NASA Glenn Research Center, Cleveland) will discuss the systematic evaluation of ferroelectric BSTO thin films driven by a specific tunable microwave application. Other sessions will cover materials, microstructure, characterization, and applications.
Microelectromechanical systems (MEMS) (Symposium EE) are the focus of significant research efforts for various microsensor and microactuator applications. While the field is primarily silicon-based, other materials are under active consideration, including metals, diamond, and silicon carbide. The symposium starts with a tutorial, including a discussion on current "hot topics" in the field, including bioMEMS and micro-optics. Sessions will highlight metrology, tribology, mechanical properties, packaging, processing techniques, and optical microsystems.

\section{Special Events}

The Plenary Speaker will be Nobel Laureate Pierre-Gilles de Gennes (College de France, ESPCI), presenting a talk on soft matter in research and industry on Monday, November 27, 6:00 p.m., Grand Ballroom, Sheraton Boston.

The Awards Ceremony will convene on Wednesday, November 29, 6:00 p.m., Grand Ballroom, Sheraton Boston. George M. Whitesides (Harvard University) will receive the Von Hippel Award, Anthony G. Evans (Princeton University) will be honored as the David Turnbull Lecturer, and Dieter M. Gruen (Argonne National Laboratory) and S.I. Stupp (Northwestern University) will each receive an MRS Medal. The MRS Medalists and the Turnbull Lecturer will present their talks as part of the series within Symposium X.

\section{Additional Events}

Representatives from various U.S. departments and agencies will hold seminars on materials-research support in the Air Force Office of Scientific Research, the Department of Energy, and the National Science Foundation. The times and locations will be listed in the Meeting Guide.

Symposium $X$ talks, presented between noon and 1:30 p.m., Monday through Thursday, include topics on materials aspects of forensics, micromachines, and biomaterials research.

Poster sessions will be held Monday through Thursday, beginning at 8:00 p.m. in the Hynes Convention Center, second level. The Meeting Chairs will sponsor a Best Poster Award Competition, awarding a prize of $\$ 500$ to the presenting author(s) of the winning paper(s). Award recipients will be selected on the basis of the poster's technical content, appearance, graphic excellence, and presentation quality.

Five Symposium Tutorial sessions by leading experts will be given on Sunday and Monday. See subsequent pages for more information.
More than 225 exhibitors will display a full spectrum of products and services at the Exhibit held from Tuesday through Thursday in the Hynes Convention Center. Research Tools Seminars, a new educational seminar series that focuses on the scientific basis and practical application of commercially available, state-ofthe-art tools, will debut this fall. Look for details in the Meeting Guide.

Information on Materials MicroWorld, a traveling program of interactive educational displays, will be on view at the Hynes Convention Center.

\section{Student Opportunities and Employment}

The Society will present Gold and Silver Graduate Student Awards to graduate students who authored or coauthored symposium papers that exemplify significant and timely research. On Wednesday evening, all finalists will be honored at the Awards Ceremony.

Graduate students who are interested in assisting in the symposium rooms are encouraged to apply for a Symposium Assistant position. Symposium assistants deliver essential meeting materials to and from the technical sessions, operate audiovisual equipment and room lighting, and perform other tasks requested by their session chairs. Symposium Assistants will receive a complimentary student registration, a one-year MRS student membership commencing January 1, 2001, and a stipend to help defray expenses.

Graduate students and members of MRS University Chapters are invited to attend the Student Mixer reception. Also, Chapter officers and faculty advisors are invited to attend a meeting of MRS University Chapter representatives to compare notes on recent activities and brainstorm on new projects and issues of common concern. Those interested in starting a new chapter are also welcome. The date, time, and location of both events will be announced in the Meeting Guide on site.

MRS, in conjunction with the American Institute of Physics (AIP), will host an Employment Center for meeting attendees. The center will operate Tuesday through Thursday, in the exhibit area on the second level of the Hynes Convention Center. Services include access to current job postings, a resume file for prospective employers, and on-site interview opportunities.

For further information on the Meeting, see the following pages and access the MRS web site at www.mrs.org. 